\title{
Image-Based Evaluation of Vascular Function and Hemodynamics
}

\author{
Jongmin Lee
}

Department of Radiology, Kyungpook National University Hospital, Daegu, South Korea

\section{Key Words}

Flow velocity . Flow volume $\cdot$ Peripheral vascular resistance $\cdot$ Wall shear stress $\cdot$ Arterial wall stiffness

\begin{abstract}
The noticeable characteristics of the blood vascular structure are the inconsistent viscosity of blood and the stiffness of the vascular wall. If we can control these two factors, we can solve more problems related to hemodynamics and vascular wall function. Understanding the properties of hemodynamics and vascular wall function may provide more information applicable to clinical practice for cardiovascular disease. The bedside techniques evaluating vascular function usually measure indirect parameters. In contrast, some medical imaging techniques provide clear and direct depictions of functional cardiovascular characteristics. In this review, image-based evaluation of hemodynamic and vascular wall functions is discussed from the perspective of blood flow velocity, flow volume, flow pattern, peripheral vascular resistance, intraluminal pressure, vascular wall stress, and wall stiffness.

(c) 2013 S. Karger AG, Basel
\end{abstract}

\section{Introduction}

The function of the vascular structure can be divided into two parts: hemodynamic function and vascular wall function. There are specific characteristics for the hemodynamic function in the human body. The most remarkable property of hemodynamics is the pulsatile flow. The pulsatile blood flow starts from the periodical ejection of blood from the left ventricle. Through the aorta and arteries, the pulsatility attenuates the blood flow, and an almost steady flow reaches the capillary level. Due to the pulsatile property of the human arterial flow, there are limitations for applying the knowledge of basic steady flow dynamics to the blood flow directly [1]. The property of blood is a factor of hemodynamics in vivo. Human blood is regarded 
as non-Newtonian fluid. Newton's law of viscosity explains the relationship among the vascular wall shear stress, the wall shear rate, and the viscosity of blood. When the viscosity is constant, the wall shear rate and stress should have a linear relationship. This kind of fluid is described as a Newtonian fluid. The viscosity of blood, a non-Newtonian fluid, changes due to the flow environment since the blood is a mixture of diverse components that interact with each other and the flow environment [2]. By decreasing the size of the blood vessel and thereby the blood flow, blood cells have a tendency to gather in the center of the flow stream, a phenomenon which is called the Fahraeus-Lindqvist effect. The eventual viscosity of the flowing blood drastically decreases as the vessel size decreases nonlinearly. This effect is reported to be evident in vessel diameters smaller than $400 \mu \mathrm{m}$. Due to this effect, the microcirculation of the blood can be facilitated with less friction on the vascular wall [3]. The geometry of blood vessels adds a specific property to the human blood flow. During the flow, the laminar flow pattern cannot be maintained due to the subsequent branching of blood vessels. The disturbed blood flow including turbulence and vortex may influence the final property of the blood flow [4].

The function of the vascular wall is complicated as is the hemodynamic function. The vascular wall has characteristics of elasticity and remodeling by intravascular and perivascular environments. The arrangement of endothelial cells is concordant to the direction of the blood flow. The blood flow and endothelial cells interact induced by friction and cellular impact. Wall shear stress is a representative marker for friction from the blood flow. According to the change in wall shear stress, the endothelial cell lining adjusts its cellular arrangement, and, subsequently, vascular pathogenesis can be ignited [5]. The media layer of the vascular wall has a dual function, namely flexibility and support. From the remodeling process, the stiffness and tolerance to inner tangential stress changes within the vascular wall. There are many indicators for vascular wall stiffness and tangential wall stress in the literature. A specific property of the vascular wall regarding its stiffness is the nonlinear, somehow sigmoidal relationship between the intravascular volume and transmural pressure [6]. Due to this fact, vascular wall elasticity can be influenced by the degree of vascular dilatation, and the value for classic vascular compliance changes along the intraluminal pressure change. Based on the Bernoulli principle, the total energy of the blood flow should consist of intrinsic, kinetic, and potential energies. The intrinsic and potential energies can be observed as the diastolic blood pressure or tangential wall stress, whereas the kinetic energy of the blood flow can be evaluated with the wall shear stress or its derivatives [7].

The noticeable characteristics of the blood vascular structure are the inconsistent viscosity of blood and the stiffness of the vascular wall. If we can control these two factors, we can solve more problems related to hemodynamic and vascular wall function. Understanding the properties of hemodynamics and vascular wall function may provide more information applicable to clinical practice for cardiovascular disease. The bedside techniques for evaluating vascular function usually measure indirect parameters. In contrast, some medical imaging techniques provide clear and direct depictions of functional cardiovascular characteristics. In this review, image-based evaluation of hemodynamic and vascular wall functions is discussed.

\section{Flow Velocity}

Since Doppler ultrasonography and magnetic resonance phase-contrast (MRPC) imaging can acquire images containing velocity information, velocity is regarded as the most important and fundamental marker representing the kinetic energy of the blood flow. The moving velocity of the ultrasound reflectors can be calculated by the Doppler equation (Equation 1). The velocity of blood cells is observed as the frequency shift of input ultrasound while receiving the reflected wave. By plotting the velocity of the ultrasound reflectors along time, 
Fig. 1. Doppler ultrasonography. a Color Doppler ultrasonography consisting of a B-mode ultrasonographic image and an overlaid color map of velocity. Incoming and outgoing flows can be mapped as red or blue colors. A higher velocity shows more extreme colors on the color bar. b By locating a sample volume within the color Doppler zone, the real-time continuous time-velocity spectrum can be acquired. White dots within the spectrum indicate velocities of each ultrasonic reflector, blood cells. By numerical calculation of the quantitative data, timeaveraged mean velocity (TAM) and other velocity derivatives can be acquired. PSV = Peak systolic velocity; EDV = end-diastolic velocity; HR = heart rate. Colors refer to the online version only.
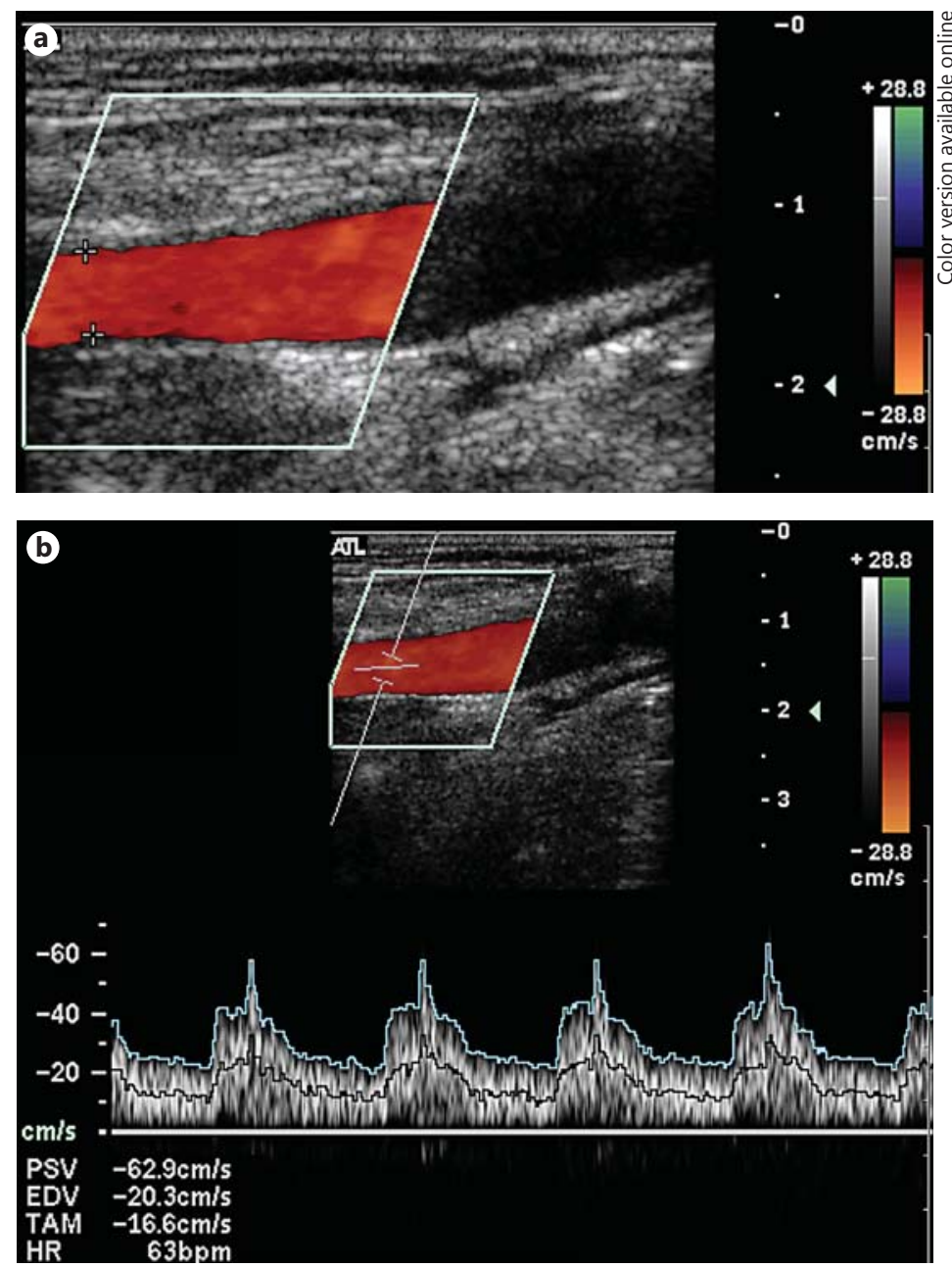

a time-velocity spectrum can be acquired. The spectrum shows the pulsatile flow pattern when it is acquired in the aorta or artery. In this spectrum, peak systolic, end-diastolic, and time-averaged mean velocities can be measured quantitatively [8] (fig. 1).

$$
\Delta f=\frac{2 f f \cdot v \cdot \cos \theta}{c},
$$

where $\Delta f$ is the frequency shift, $f$ the frequency of ultrasound, $v$ the velocity, $\theta$ the ultrasound entry angle, and $c$ the velocity of the ultrasound in the media.

The MRPC image can be acquired by adding a bipolar gradient ahead of the routine gradient echo sequence. Due to the bipolar pretreatment, the MR signal from static tissue does not change in contrast to the altered signal in moving protons within the blood flow. Subtracting the image with the baseline mask image, only the flow is displayed. In the phase image, the signal intensity suggests a flow velocity with a linear relationship (Equation 2) (fig. 2). During MRPC imaging, several decades of phase images are reconstructed by time phases. During the subsequent postprocessing, the time-velocity curve can be plotted:

$$
v=V_{e n c} \cdot \frac{I}{\pi},
$$

where $v$ is the velocity, $V_{\text {enc }}$ the velocity encoding value, and $I$ the signal intensity. 

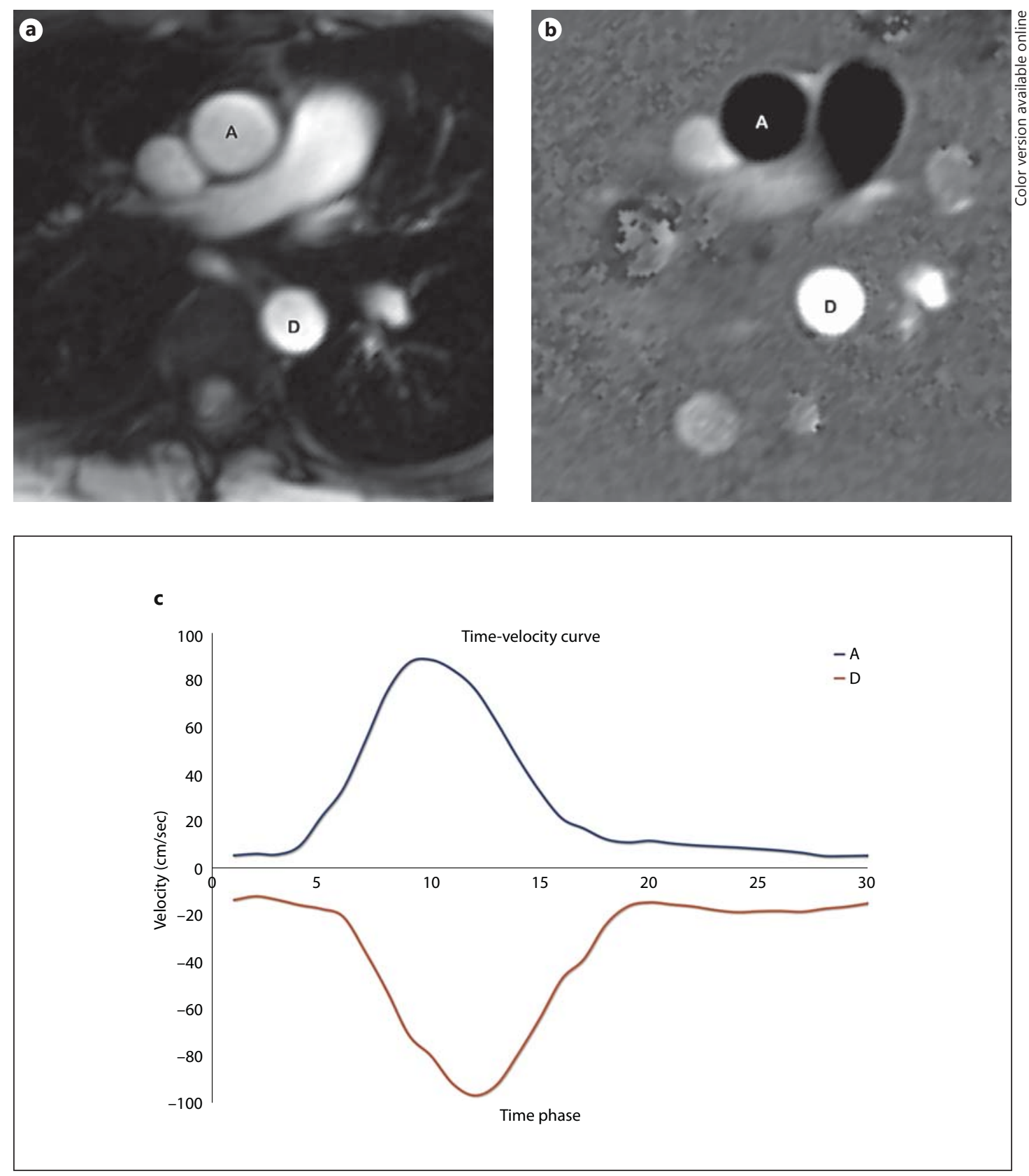

Fig. 2. Velocity-encoded MRPC imaging. a Basic fast gradient-echo image (magnitude image) can be acquired in high temporal resolution and can be seen as a cine image. With this image, the anatomical location of the blood flow can be identified. Setting ROIs can be done on this magnitude image since the edge detection is easier than in a phase image. $\mathbf{b}$ By adding an initial bipolar pulse on the fast gradient-echo sequence, protons within the static tissue and dynamic flow should show the nulled and bipolar phases of precession. The difference in phase implies the velocity of the proton, which is displayed in grey scale by its direction across the imaging plane. Ascending and descending aortas show opposite grey scale since the flow direction is opposite. $\mathbf{c}$ By locating the ROI on the magnitude image, the flow velocity data can be acquired from the phase image. Although they are not in real time, time-velocity curves can be drawn by post-processing the acquired data. The descending aortic flow shows a negative value by the opposite flow direction to the ascending aortic flow. A = Ascending aorta; $\mathrm{D}=$ descending aorta. 
In MR processing, a single average velocity from a region of interest (ROI) can be displayed, while the Doppler ultrasonographic spectrum plots all the velocity information from the reflectors. Another difference between MRPC imaging and Doppler ultrasonography is that the time-velocity curve in MRPC images is for one cardiac cycle, which is assembled from several fragmented information during multiple cardiac cycles. In contrast, Doppler ultrasonography displays a time-velocity spectrum acquired in real time. In addition, MRPC imaging possesses potential errors from mismatched encoding velocity, deviated imaging plane, spatial misregistration of images, and so on [9]. To overcome these drawbacks of MRPC imaging, not only strict management of scanning skills is carried out but also further modification of the velocity calculation formula [10].

Regardless of the inherent potential errors of MRPC imaging, this technique has become popular for in vivo blood flow evaluation. Kim et al. [10] reported the in vitro validation of MRPC performance using a self-made pulsatile flow system and self-modified velocimetry formula (Equation 3). The basic formula for velocimetry (Equation 2) was standardized with the background noise and signal intensity of the magnitude image. The correlation between the MRPC velocity and the physically measured velocity by a mechanical flow meter was significantly high $\left(R^{2}=0.848\right)$ in vitro. The in vivo validation was already reported in 1987 by Firmin et al. [11]. The MRPC-derived ascending aortic flow volume was compared with the left ventricular stroke volume measured from a cine image, and the correlation was reported to be significantly high ( $r=0.97)$. Recently, the three-dimensional (3D) MRPC technique was validated based on Doppler ultrasonography. In a very well-organized experiment using dogs, Jiang et al. [12] compared the velocity waveforms and peak systolic velocities obtained from Doppler ultrasonography and 3D MRPC images and reported a significantly good correlation ( $\mathrm{r}=0.89$ and $\mathrm{r}=0.83$, respectively).

$$
v=a \cdot \frac{V_{e n c}}{\pi} \cdot \frac{I_{p}}{I_{m}},
$$

where $v$ is the velocity, $a$ the correction coefficient, $V_{\text {enc }}$ the velocity encoding value, $I$ the signal intensity, $p$ the phase image, and $m$ the magnitude image.

\section{Flow Volume}

With Doppler ultrasonography or MRPC imaging, time-velocity spectrum or plotting can be obtained as fundamental hemodynamic quantitative information. From the velocity value, the second derivatives can be acquired. Volume flow per unit time (flow volume, $\mathrm{ml} / \mathrm{s}$ ) is one of the popular secondary markers derived from velocity. By measuring the cross-sectional area or diameter of the blood vessel either in real time or through one cycle of pulsatile flow, the velocity can be multiplied by another geometric dimension to calculate the flow volume. In the time-velocity spectrum of Doppler ultrasonography, the time-averaged mean velocity can be obtained in real time. In M-mode ultrasonography at the center of the blood vessel, the pulsatile variation of the luminal diameter can be measured to calculate the time-averaged mean area or exact luminal area at each time phase. In the case of MRI, the MRPC image shows the mean flow velocity within the ROI, of which the size is the cross-sectional area of the flow.

The flow volume is popular as an indicator for the amount of blood flow or tissue perfusion at a target organ. If the blood flow measurement is applied to the cerebral arteries - the two internal carotid and two vertebral arteries - the total cerebral circulation volume and net distribution of cerebral blood flow can be evaluated. The evaluation of the net cerebral blood flow can analyze the compensatory interaction among the cerebral arteries and show blood flow deficits unrelated to morphological arterial stenosis (fig. 3). In addition, by including the 
Pulse

Fig. 3. Flow volume analysis in cerebral arteries of a 70-year-old asymptomatic male during a health screening. a By using the flow diameter and time-averaged mean flow velocity (TAM), the volume flow can be estimated. The left internal carotid artery shows a normal flow pattern and velocities. b The left internal carotid artery is completely occluded showing no flow and a calcified plaque within the lumen. c The left vertebral artery shows a normal flow pattern and velocities. Due to cardiac arrhythmia, the time-velocity spectrum shows irregular pulse intervals. d The right vertebral artery is completely occluded without any flow signal. e A comprehensive cerebral blood flow analysis chart displays the flow volume of the bilateral internal and external carotid and vertebral arteries. In this patient, the right internal carotid and vertebral arteries show no flow (green rectangles). The trace values are due to wall vibration artifacts. However, the total cerebral blood flow volume is within the normal range due to a compensatory overflow in the left internal carotid artery (red rectangle). PSV = Peak systolic velocity; $\mathrm{EDV}=$ end-diastolic velocity; $\mathrm{HR}=$ heart rate. Colors refer to the online version only.

\begin{tabular}{l|l}
\hline Pulse 2013;1:108-122 \\
\hline DOI: $10.1159 / 000354110$ & $\begin{array}{l}\text { @ } 2013 \text { S. Karger AG, Basel } \\
\text { www.karger.com/pls }\end{array}$ \\
\hline
\end{tabular}

Lee: Image-Based Evaluation of Vascular Function and Hemodynamics
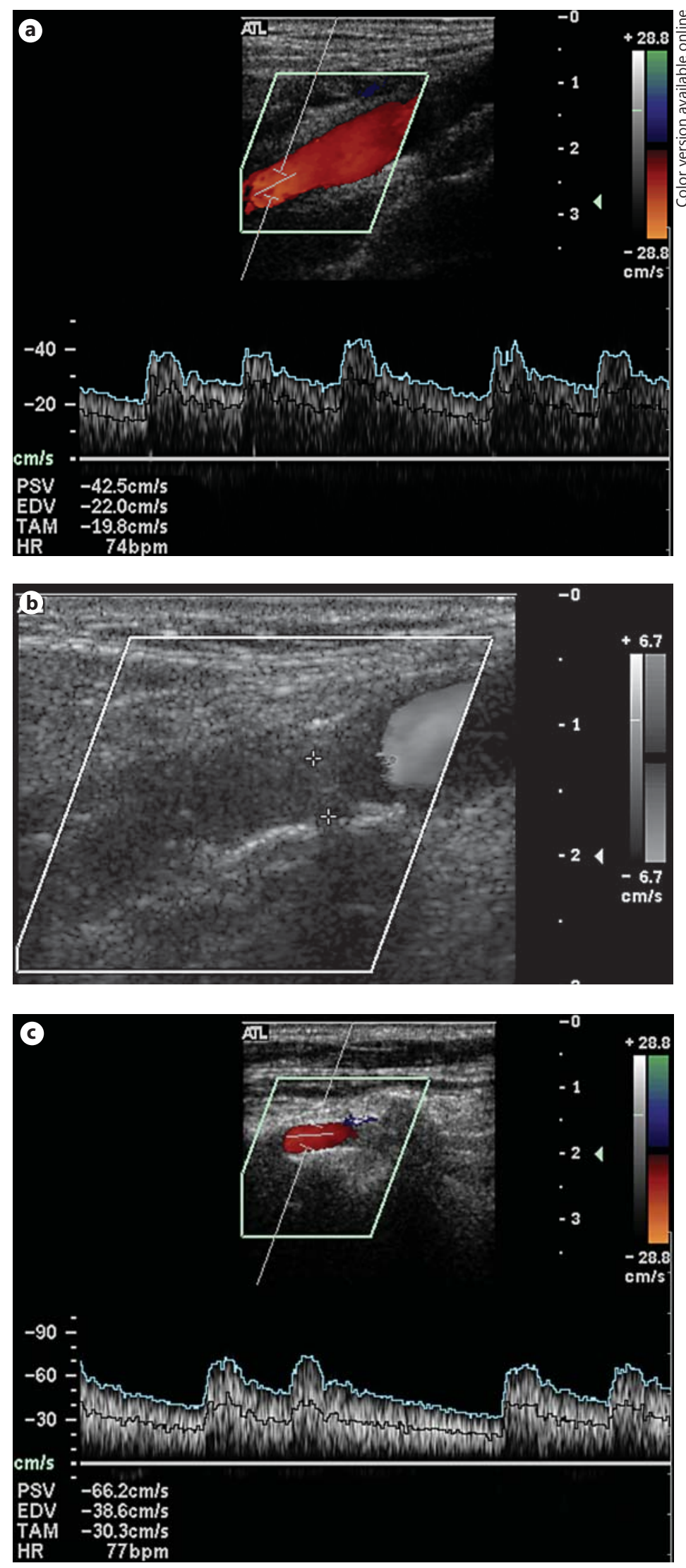


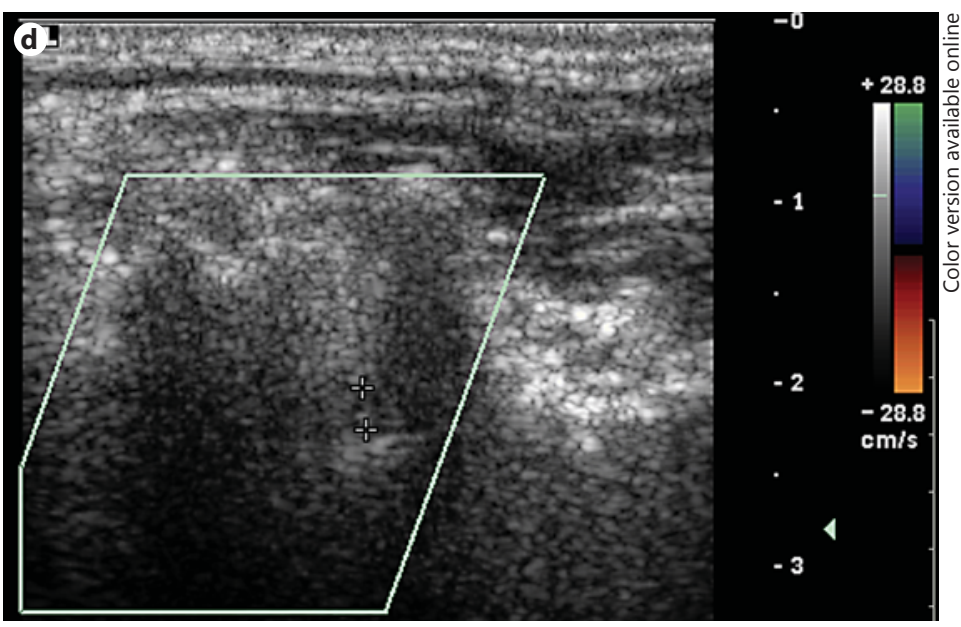

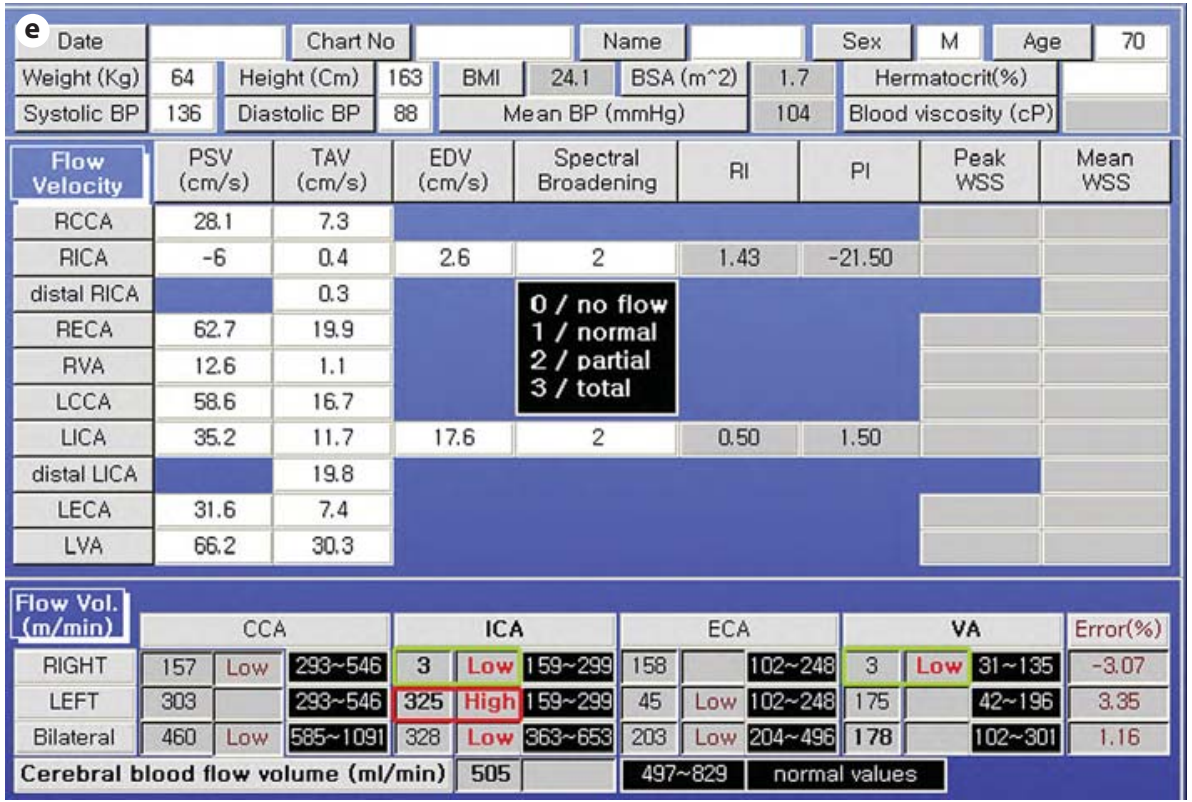

(For legend see p. 113.)

external carotid arteries in the flow measure protocol, an internal validation of the technique is possible. The sum of the bilateral internal and external carotid arterial flow should approach the sum of the bilateral common carotid arterial flow. Using Doppler four-vessel flowmetry in a cohort of patients with acute cerebral stroke, Jung et al. [13] reported that the contralateral internal carotid arterial, anterior cerebral, and total cerebral blood flow volumes were statistically significant markers to predict cerebral vascular reserve.

While ultrasonographic flowmetry is confined to ultrasonographically accessible rather superficial arteries, MRPC imaging is not restricted by the location of the target artery. Especially for mediastinal great vessels, MRPC is a good choice for flowmetry. By directly measuring the flow volume in the aorta and pulmonary artery, the Qp/Qs can be calculated easily if there is no semilunar valvular regurgitation. In addition, by measuring the flow volume of the pulmonary artery branches, the regional distribution of the pulmonary arterial flow can be analyzed. With a to-and-pro flow pattern in the great vessels, especially in cases with congenital heart disease, the net forward flow volume to a target organ can be calculated. The 
MRPC flowmetry technique can be validated within a subject by comparing the gap between the ascending and descending aortic flow volume with the superior vena cava flow volume [14].

\section{Flow Pattern}

Since arterial and major venous flows are pulsatile due to ventricular contraction and respiration, the pattern of flow pulsatility yields more information about the hemodynamic and vascular morphological status. One of the useful pieces of information from flow pattern analysis is the hemodynamic influence of a stenotic segment. In a pre-stenotic segment, blood flow experiences a higher flow resistance so that the peak systolic and end-diastolic velocities have a great difference (high impedance flow). At a stenotic segment, flow velocity increases in a reverse correlation with the reduction in the luminal area. Usually, the high impedance flow pattern is preserved although a disturbed flow may occur. In a post-stenotic segment, the flow energy decreases due to wall shear and flow disturbance through the stenotic segment. This flow attenuation can be seen as a decreased peak systolic velocity with a relatively high diastolic velocity (low impedance flow). In the case of severe stenosis, extreme flow attenuation can exhibit a damped flow spectrum with delayed pulses (parvus and tardus) [15]. Based on the Bernoulli principle, the stenosis degree can be determined from the ratio of the flow velocities between the pre-stenotic and stenotic segments (Equation 4). If measuring the stenotic flow is limited due to high flow artifacts or vibrations, the stenotic jet just distal to the stenosis can reflect the flow status at the stenotic segment. The stenosis measured by flow alteration can be called 'hemodynamic stenosis', which has the basic assumption that there is no energy loss at the stenotic segment.

$$
\text { Diameter stenosis }(\%)=100 e\left(11 \sqrt{\frac{v_{1}}{v_{2}}}\right) \text {, }
$$

where $v_{1}$ is the prestenotic flow velocity and $v_{2}$ the stenotic flow velocity.

Another noticeable point in the flow pattern is the spectral window. The spectral window refers to clear windows at the bottom of the flow-velocity spectrum acquired from Doppler ultrasonography. Since the flow-velocity spectrum plots whole signals from the reflectors, the bright spots indicate the flow velocity of the reflector. If the velocity profile is a plug or bullet shaped with a laminar pattern, the spectral window may become wide and the spectral line becomes thin. In contrast, if the velocity of the reflectors is heterogeneous with a very slow bullet or disturbed flow, the spectral line may become thick and the spectral window should become narrow. The relative size or conspicuity of the spectral window suggests the probability of a disturbed flow within the target vessel.

To analyze flow patterns of major arteries, normal features should be acknowledged as well. Cerebral, renal, and hepatosplenic arteries have a relatively constant lumen without remarkable vasoactivity compared to gastrointestinal or muscular arteries. Constant arteries exhibit a relatively low impedance flow with a preserved diastolic flow volume, while muscular arteries exhibit a high impedance flow with almost zero diastolic flow at rest. In the extremity arteries, due to a strong recoil function of the elastic arterial wall, a biphasic or triphasic flow pattern is normally observed. An abnormally high impedance flow may suggest distal stenosis or a stiff wall. An abnormally effaced multiphasic flow may suggest either exertion-related active vasodilatation or a stiff and dilated wall mostly due to diabetic vasculopathy. 
Lee: Image-Based Evaluation of Vascular Function and Hemodynamics

\section{Flow Resistance}

Peripheral vascular resistance may be the most difficult parameter to evaluate using noninvasive modalities. However, due to the pulsatility of the arterial flow, we can approximate the distal flow resistance indirectly. The resistance index (RI) is a popular marker during Doppler ultrasonographic examination of the blood flow (Equation 5). In visceral arteries such as the uterine and renal arteries, the normal RI value ranges from 0.5 to 0.8 . Higher RI values may suggest insane parenchymal capillary flow in the target organ. Although RI is a widely used marker for vascular resistance, a limitation is that a pseudonormal result is possible when systolic and diastolic velocities increase simultaneously. To overcome this limitation, the pulsatility index is suggested by standardization of the flow velocity (Equation 6) [16].

$$
R I=\frac{(P S V-E D V)}{P S V}
$$

where $P S V$ is the peak systolic velocity and $E D V$ the end-diastolic velocity.

$$
P I=\frac{(P S V-E D V)}{T A M},
$$

where $P I$ is the pulsatility index and TAM the time-averaged mean velocity.

Theoretically, flow resistance can be evaluated based on the Poiseuille equation (Equation 7a). The basic principle to measure flow resistance is based on Ohm's law. If the flow volume and intraluminal pressure gradient are acquired, flow resistance can be easily calculated (Equation $7 \mathrm{~b}$ ). However, the prerequisites for this calculation are a straight rigid tube with a thin wall and a steady flow of a Newtonian fluid. These conditions are not acceptable for the human blood flow environment [1]. As we already discussed, MRPC imaging or Doppler ultrasonography can measure the flow volume noninvasively and reliably. Muthurangu et al. [17] measured pulmonary arterial resistance using MRPC imaging with the help of invasive intraluminal manometry and reported noticeable results.

$$
\begin{aligned}
& Q=\frac{\pi \cdot r^{4} \cdot \Delta p}{8 \Delta \mu \cdot L} \text { and } \\
& R=\frac{\Delta p}{Q}=\frac{8 \cdot \mu \cdot L}{\pi \cdot r^{4}},
\end{aligned}
$$

where $Q$ is the flow volume, $\Delta p$ the pressure gradient, $R$ the flow resistance, $\mu$ the fluid viscosity, $L$ the length of the tube, and $r$ the radius of the tube.

\section{Intravascular Pressure}

Since measuring the absolute pressure value is impossible by using noninvasive imagebased methods, the pressure gradient measurement is an alternative choice to evaluate the pressure-related hemodynamic function. During the routine echocardiographic evaluation of the stenotic or transvalvular flow, the pressure gradient measurement is displayed automatically if the flow velocity is acquired. The rationale of this measurement is the simplified Bernoulli equation (Equation 8) [18]:

$$
\Delta p=4 \mathrm{Y} V^{2},
$$


where $\Delta p$ is the pressure gradient (in $\mathrm{mm} \mathrm{Hg}$ ) and $V$ is the peak systolic velocity (in $\mathrm{m} / \mathrm{s}$ ).

$$
E_{t}=P_{1}+\frac{1}{2} \cdot \rho \cdot v_{1}^{2}+\rho \cdot g \cdot h_{1}=P_{2}+\frac{1}{2} \cdot \rho \cdot v_{2}^{2}+\rho \cdot g \cdot h_{2},
$$

where $E_{t}$ is the total flow energy, $P$ the static pressure, $\rho$ the blood density, $v$ the flow velocity, $g$ the gravitational acceleration, and $h$ the height of the blood vessel; 1 indicates the prestenotic level of the blood vessel, and 2 denotes the stenotic segment.

The Bernoulli principle is the law of flow energy (momentum) conservation (Equation 9). Although there is the assumption that no energy is lost by friction, this formula explains the relationship among intrinsic, kinetic, potential, and total energies of the intravascular flow. If pre-stenotic and stenotic segments of blood vessels are at the same height against gravity, if the pre-stenotic flow velocity is negligible to zero, and if the pressure in the stenotic segment is the same as the post-stenotic pressure, the Bernoulli equation can be simplified as Equation 8. As shown here, the simplified Bernoulli equation is based on many significant assumptions, which may be important to maintain the accuracy and reproducibility of the trans-stenotic pressure gradient measurement. Actually, the simplified Bernoulli equation is a function of the stenotic flow velocity only. The stenotic flow velocity may be influenced by the real trans-stenotic pressure gradient but may not be a direct marker for the pressure gradient. The difference in velocities between the pre-stenotic and stenotic segments is the difference in the kinetic energy, which should be compensated by the intrinsic energy (static pressure) to conserve the total energy of the blood flow. In other words, the stenotic flow velocity is just an altered proportion of the kinetic energy among the total flow energy and not a pressure difference provoked by the stenosis. This fact is an inherent limitation of the simplified Bernoulli equation during pressure gradient evaluation [18].

Although a nondisturbed Newtonian fluid flow was an assumption, Thompson and McVeigh [19] measured the intracardiac pressure gradient using the Navier-Stokes equation, which is a 3D set of nonlinear equations for the conservation of momentum of a fluid (Equation 10a). They derived a sophisticated equation for the pressure gradient between adjacent elements based on the Navier-Stokes equation with neglecting friction and gravity terms. Using the finite element analysis technique, the equation was simplified as a function of velocity and element geometry (Equation 10b). Based on reference standards from intraluminal pressure transducers in vitro and in vivo, the authors reported excellent validation results of their own MRPC-based pressure gradient measurement technique.

$$
\begin{aligned}
& -\frac{\partial P}{\partial x_{i}}=\rho \frac{\partial v_{i}}{\partial t}+\rho\left[v_{1} \frac{\partial v_{i}}{\partial x_{1}}+v_{2} \frac{\partial v_{i}}{\partial x_{2}}+v_{3} \frac{\partial v_{i}}{\partial x_{3}}\right]-\mu\left[\frac{\partial^{2} v_{i}}{\partial x_{1}^{2}}+\frac{\partial^{2} v_{i}}{\partial x_{2}^{2}}+\frac{\partial^{2} v_{i}}{\partial x_{3}^{2}}\right]-F_{i}, i \text { and } \\
& \Delta P=\sum_{i}\left[\left(\frac{\partial P}{\partial x}\right)_{i} \Delta x_{i}+\left(\frac{\partial P}{\partial y}\right)_{i} \Delta y_{i}\right]
\end{aligned}
$$

where $x_{i}^{\prime}$ s are the $\mathrm{x}^{-}, \mathrm{y}-$, and $\mathrm{z}$-axes in the image frame, $v$ is the velocity, $\mu$ the coefficient of the viscosity, $\rho$ the fluid density, $P$ the pressure, and $F$ the gravitational force.

Using the Navier-Stokes equation, Lum et al. [20] validated the 3D MRPC-based pressure gradient measurement technique. Based on the data acquired from endovascular pressuresensing guidewires, pressure gradient mapping was conducted on the carotid, renal, and iliac arteries of swine. The correlation coefficient was 0.952 with statistical significance.

Lee et al. [21] introduced an MRPC-based pressure gradient evaluation technique as a function of the stenosis degree, stenotic length, and input velocity. The relationships between the intraluminal pressure and the three functional parameters were analyzed in a steady flow 
system in vitro. With a mechanically measured trans-stenotic pressure gradient, the three parameters showed a linear correlation. By integrating all the relationships, a morphofunctional formula for the pressure gradient was suggested and validated in vitro $(r=0.9977, \mathrm{p}<$ 0.0001).

\section{Endovascular Wall Shear Stress}

The function of the vascular wall may consist of different kinds of reactions against blood and its flow. The boundary of the blood flow is the flow-tissue interface, where the endothelial cell lining is exposed to flow-induced friction. This friction-provoking energy can be evaluated with the wall shear rate and wall shear stress (Equation 11). The wall shear rate can be acquired from the velocity profile drawn by a polynomial interpolation of the velocity data along the perpendicular geometry. The gradient of the outermost location of the bolus geometry is the wall shear rate [22].

$$
\gamma\left(\frac{d u}{d y}\right) \text { and }
$$

$\tau$,

where $\gamma$ is the wall shear rate, $u$ the flow velocity, $y$ the distance from the wall, and $\tau$ the wall shear stress.

Wall shear stress influences the microstructure of the endothelial lining of blood vessels. Under a steadily high stress, endothelial cells remodel to fortify their tight junctions. A low or oscillating wall shear stress loosens the tight junctions and widens the extracellular space. Through the loose tissue, the penetration of atherogens becomes easier, and subsequently more atherosclerotic plaque can be generated. At the outer side of branching vessels or the inner side of curved vessels, there usually exists a low or oscillating wall shear stress and, subsequently, a higher probability of atherosclerotic plaque formation. This is the rationale for the higher prevalence of atherosclerotic plaque in the outer wall of the carotid sinus, where flow separation occurs during diastole [23].

Kim et al. [10] validated the MRPC-based wall shear stress evaluation technique using a steady laminar flow model. With the mechanically measured mean shear stress as a function of the pressure gradient, diameter, and measuring distance, the MRPC-based wall shear stress showed a good correlation $\left(\mathrm{R}^{2}=0.954, \mathrm{p}<0.001\right)$. In a curved tube, the authors could depict significantly different wall shear stresses between the inner and outer sides.

Since oscillatory wall shear stress provokes an early change in atherosclerosis in the exposed endothelial layer, the oscillatory shear index is a promising secondary marker for the real impact of wall shear stress (Equation 12) [24]. A higher oscillatory shear index suggests more fluctuation of the wall shear stress vector from the main stream during pulsatile blood flow. Isoda et al. [25] evaluated wall shear stress and the oscillatory shear index in cerebral arterial aneurysms using the 3D MRPC technique. They discovered that at least one spiral flow existed within each aneurysm, and the aneurysmal apex, which is usually vulnerable to rupture, presented a higher oscillatory shear index.

$$
O S I=\frac{1}{2}\left(1-\frac{\left|\int_{0}^{\tau} \tau_{i} d t\right|}{\int_{0}^{\tau}\left|\tau_{i}\right| d t}\right),
$$

where OSI is the oscillatory shear index and $\tau$ the wall shear stress. 


\section{Tangential Wall Stress}

Tangential stress is a stress of the vascular wall against the perpendicular internal pressure generated by the contained blood. This tangential stress can be explained by the law of Laplace (Equation 13). One example of the tangential stress is the left ventricle. The basal and apical levels normally have thicker and thinner walls than that of each other. Since the intrinsic pressure of the left ventricle is the same across the level, the larger radius requires a thicker wall to conserve the constant tangential stress [26].

$$
W_{s}=\frac{P \cdot R}{k \cdot h}
$$

where $W_{s}$ is the wall stress, $k$ the constant, $P$ the intravascular pressure, $h$ the wall thickness, and $R$ the radius.

The concept of tangential wall stress can be adopted when evaluating the risk of aortic aneurysms. Using the aneurysmal diameter and wall thickness from CT images together with brachial blood pressure from a cuff manometer, Lee et al. [27] suggested the tangential stress index for the prediction of the risk for rupture of an aortic aneurysm. The index was the ratio between the measured tangential wall stress and the ideal value based on normal aortic geometry and blood pressures. These authors reported a significant difference in the index value between the normal, unruptured, and ruptured aneurysm groups $(\mathrm{p}<0.01)$. Using a cutoff value for the diastolic tangential stress index, they reached $100 \%$ sensitivity and $88 \%$ specificity to differentiate a ruptured aneurysm from a stable one.

Di Martino et al. [28] compared ruptured and stable abdominal aortic aneurysms using tissue specimens. The classic predictor for aneurysmal rupture, the diameter, showed no difference between the cohorts. Instead, the wall thickness and ultimate tensile strength demonstrated a significant difference and did not correlate with the diameter. The authors suggested that measuring the wall thickness or stiffness might be a better predictor of rupture for large abdominal aortic aneurysms.

\section{Vascular Wall Stiffness}

One of the most important roles of major arteries is the damping of the pulsatile flow energy to maintain continuous blood flow and to reduce the hemodynamic stress in smaller arteries at end organs. To fulfill this role, the arterial wall has the property of elasticity. Closer to the heart, the aortic wall possesses a greater proportion of elastin to achieve higher elasticity. The elastic function of the aortic and arterial walls can be evaluated using several markers. The most widely used classical marker for arterial wall elasticity is compliance (Equation 14). Compliance is simple and easy to apply in practice. However, since the pressure-volume curve is nonlinear, the compliance value changes according to the diastolic intraluminal pressure. An accurate evaluation of wall elasticity using compliance is limited in vivo [29].

$$
C=\frac{\Delta V}{\Delta P},
$$

where $C$ is the compliance, $V$ the intravascular volume, and $P$ the intraluminal pressure.

To compensate for the limitation of compliance, Young's modulus of elasticity was suggested (Equation 15a). This index measures the stiffness of isotropic elastic material as the ratio between stress and strain at the unit area. To apply Young's modulus of elasticity on the cardiovascular system, a secondary derivative called the stiffness index $(\beta)$ was intro- 
duced (Equation 15b). Another marker is the arterial distensibility, which is nowadays widely used to analyze the arterial wall stiffness (Equation 16). These methods modified classical compliance by normalizing the static geometry of the vascular lumen and vascular wall thickness. Recently, the arterial wall stiffness index was suggested as a function of the systolic and diastolic intraluminal pressure and volume based on data acquired from swine aorta ex vivo (Equation 17). Although the arterial wall stiffness index is not suitable for manual calculation during routine practice due to its complexity, this index recorded the best validation score in vitro and in vivo [29].

$$
\begin{aligned}
& Y=\frac{\Delta P}{\Delta D} \cdot \frac{D_{0}}{h} \text { and } \\
& \beta=\ln \left[\frac{P_{s}}{P_{d}}\right] \cdot \frac{D_{0}}{\Delta D},
\end{aligned}
$$

where $Y$ is Young's modulus of elasticity, $P$ is the intraluminal pressure, $D$ the vascular diameter, $D_{0}$ the diameter at diastole, $h$ the wall thickness, $\beta$ the stiffness index, $P_{s}$ the systolic pressure, and $P_{d}$ the diastolic pressure.

$$
D=\frac{\Delta A}{A_{0} \cdot \Delta P}
$$

where $D$ is the arterial distensibility, $A$ the cross-sectional area of the vascular lumen, $A_{0}$ the diastolic cross-sectional area, and $P$ the intraluminal pressure.

$$
\begin{aligned}
& a=\sqrt{\frac{V_{d} \cdot P_{d}^{2}-V_{s} \cdot P_{s}^{2}}{V_{s}-V_{d}}}, \\
& b=\frac{V_{d} \cdot V_{s} \cdot\left(P_{d}^{2}-P_{s}^{2}\right)}{V_{d} \cdot P_{d}^{2}-V_{s} \cdot P_{s}^{2}} \text { and } \\
& A W S I=\sqrt{\left[\operatorname { l n } \left[\frac{-2 b}{a^{2}} \cdot P_{m}\right.\right.} \frac{1}{\left\{1+\left(\frac{P_{m}}{a}\right)^{2}\right\}} \cdot \frac{\left.V_{d}\right] \mid}{[}
\end{aligned}
$$

where $d=$ diastolic, $s=$ systolic, and $m=$ mean; $V$ is the intravascular volume, $P$ the intraluminal pressure, and $A W S I$ the arterial wall stiffness index.

The arterial wall stiffness can be evaluated by indirect functional markers. In clinical routine practice, applanation tonometry to measure pulse wave velocity in superficial arteries is popular due to its simplicity of application. This technique catches arterial pulses simultaneously at distant points and measures their time gap, which can calculate the velocity of the pulse wave together with the imaginary distance. Using the Bramwell-Hill equation, the pulse wave velocity can be converted to arterial distensibility, which is a marker for arterial wall stiffness (Equation 18). Due to the wide application of the pulse wave velocity technique, validation and normal reference values were reported and acknowledged as well [30].

$$
\begin{aligned}
& v_{p w}^{2}=\frac{1}{\rho} \cdot \frac{\Delta P}{\Delta V} \cdot V_{0} \text { and } \\
& D=\frac{1}{\rho \cdot v_{p w}^{2}},
\end{aligned}
$$


where $v_{p w}$ is the pulse wave velocity, $V$ the intravascular volume, $P$ the intraluminal pressure, and $\rho$ the blood density.

Can we measure the functional marker pulse wave velocity using imaging modalities for hemodynamics? MRPC imaging has been tried already. Since velocity-encoded phase-contrast imaging is synchronized with the cardiac cycle, the time from the R-wave peak to a pulsatile flow curve can be measured and compared among multiple different imaging planes. From the MRPC images, the time-flow curve can be acquired from the ascending, intrathoracic descending, and abdominal aortas. Subsequently, the time to peak, ascending maximal slope, or foot of the curve can be measured. The gaps of these times from each aortic level can be used to calculate the pulse wave velocity between the ascending and intrathoracic descending or abdominal aortas. This MR-based pulse wave velocity is a more direct method than the conservative functional method since exact anatomical points are measured. The MR-based technique was validated as being comparable with the applanation tonometry method [31].

\section{Conclusion}

For understanding the anatomy, physiology, and pathology of the vascular system, not only the morphological properties but also the functions of blood flow and vascular wall are important. Morphological evaluation is relatively easy and popular in clinical and radiological fields. However, functional evaluation of the vascular system is now well acknowledged except for simple conservative techniques. By acquiring further clinical information from the data of hemodynamics and wall function, early diagnosis of pathological conditions and accurate monitoring of the pathophysiological processes and treatment effects could be facilitated.

\section{Acknowledgement}

This research was supported by the MSIP(Ministry of Science, ICT \& Future Planning) support program (NIPA-2013-H0301-13-2004) supervised by the NIPA (National IT Industry Promotion Agency) and the Basic Science Research Program through the National Research Foundation of Korea (NRF) funded by the Ministry of Education, Science and Technology (2010-000-7337).

\section{References}

1 Pfitzner J: Poiseuille and his law. Anaesthesia 1976;31:273-275.

- 2 Biasetti J, Hussain F, Gasser TC: Blood flow and coherent vortices in the normal and aneurysmatic aortas: a fluid dynamical approach to intra-luminal thrombus formation. J R Soc Interface 2011;8:1449-1461.

3 Azelvandre F, Oiknine C: Fahraeus effect and Fahraeus-Lindqvist effect (in French). C R Acad Sci Hebd Seances Acad Sci D 1977;284:577-580.

- 4 Chakravarty S, Sen S: Analysis of pulsatile blood flow in constricted bifurcated arteries with vorticity-stream function approach. J Med Eng Technol 2008;32:10-22.

- 5 Malek AM, Alper SL, Izumo S: Hemodynamic shear stress and its role in atherosclerosis. JAMA 1999;282: 2035-2042.

6 Drzewiecki G, Field S, Moubarak I, Li JK: Vessel growth and collapsible pressure-area relationship. Am J Physiol 1997;273:H2030-2043.

7 White FM: Fluid Mechanics, ed 7. New York, McGraw Hill, 2011.

- 8 Scoutt LM, Zawin ML, Taylor KJ: Doppler US. Part II. Clinical applications. Radiology 1990;174:309-319.

- 9 Lotz J, Meier C, Leppert A, Galanski M: Cardiovascular flow measurement with phase-contrast MR imaging: basic facts and implementation. Radiographics 2002;22:651-671.

10 Kim T, Seo JH, Bang SS, Choi HW, Chang Y, Lee J: Optimization of MR phase-contrast-based flow velocimetry and shear stress measurements. Int J Cardiovasc Imaging 2010;26(suppl 1):133-142.

-11 Firmin DN, Nayler GL, Klipstein RH, Underwood SR, Rees RS, Longmore DB: In vivo validation of MR velocity imaging. J Comput Assist Tomogr 1987;11:751-756. 
12 Jiang J, Strother C, Johnson K, Baker S, Consigny D, Wieben O, Zagzebski J: Comparison of blood velocity measurements between ultrasound Doppler and accelerated phase-contrast MR angiography in small arteries with disturbed flow. Phys Med Biol 2011;56:1755-1773.

13 Jung HY LH, Kim HJ, Baik SK, Lee J, Kim YS, Kang DS: Doppler ultrasonographic parameters for predicting cerebral vascular reserve in patients with acute ischemic stroke. J Korean Soc Ultrasound Med 2006;25:35-40.

14 Goo HW, Al-Otay A, Grosse-Wortmann L, Wu S, Macgowan CK, Yoo SJ: Phase-contrast magnetic resonance quantification of normal pulmonary venous return. J Magn Reson Imaging 2009;29:588-594.

15 Kotval PS: Doppler waveform parvus and tardus. A sign of proximal flow obstruction. J Ultrasound Med 1989; 8:435-440.

16 Takahashi K, Ohkuchi A, Hirashima C, Matsubara S, Suzuki M: Establishing reference values for mean notch depth index, pulsatility index and resistance index in the uterine artery at 16-23 weeks' gestation. J Obstet Gynaecol Res 2012;38:1275-1285.

17 Muthurangu V, Taylor A, Andriantsimiavona R, Hegde S, Miquel ME, Tulloh R, Baker E, Hill DL, Razavi RS: Novel method of quantifying pulmonary vascular resistance by use of simultaneous invasive pressure monitoring and phase-contrast magnetic resonance flow. Circulation 2004;110:826-834.

18 Heys JJ, Holyoak N, Calleja AM, Belohlavek M, Chaliki HP: Revisiting the simplified bernoulli equation. Open Biomed Eng J 2010;4:123-128.

19 Thompson RB, McVeigh ER: Fast measurement of intracardiac pressure differences with 2D breath-hold phase-contrast MRI. Magn Reson Med 2003;49:1056-1066.

20 Lum DP, Johnson KM, Paul RK, Turk AS, Consigny DW, Grinde JR, Mistretta CA, Grist TM: Transstenotic pressure gradients: measurement in swine - retrospectively ECG-gated 3D phase-contrast MR angiography versus endovascular pressure-sensing guidewires. Radiology 2007;245:751-760.

21 Lee N, Heo S, Kim J, Yoon S, Do Y, Park C, Lee J: Multivariate measurement of trans-stenotic pressure gradient. 67th Korean Congress of Radiology, Seoul, 2011, pp 382-383.

$\checkmark 22$ Cheng CP, Parker D, Taylor CA: Quantification of wall shear stress in large blood vessels using Lagrangian interpolation functions with cine phase-contrast magnetic resonance imaging. Ann Biomed Eng 2002;30: 1020-1032.

23 Shaaban AM, Duerinckx AJ: Wall shear stress and early atherosclerosis: a review. AJR Am J Roentgenol 2000; 174:1657-1665.

24 Ku DN, Giddens DP, Zarins CK, Glagov S: Pulsatile flow and atherosclerosis in the human carotid bifurcation. Positive correlation between plaque location and low oscillating shear stress. Arteriosclerosis 1985;5:293302.

25 Isoda H, Ohkura Y, Kosugi T, Hirano M, Takeda H, Hiramatsu H, Yamashita S, Takehara Y, Alley MT, Bammer R, Pelc NJ, Namba H, Sakahara H: In vivo hemodynamic analysis of intracranial aneurysms obtained by magnetic resonance fluid dynamics (MRFD) based on time-resolved three-dimensional phase-contrast MRI. Neuroradiology 2010;52:921-928.

-26 Valentinuzzi ME, Kohen AJ: Laplace's law: what it is about, where it comes from, and how it is often applied in physiology. IEEE Pulse 2011;2:74-84.

27 Lee JJ, Chang YM, Ryeom HK, Lee SK, Kim YJ, Dang DS: A suggestion of new integrated prognostic factor for aortic aneurysm: tangential stress index. J Korean Radiol Soc 1999;40:1077-1085.

-28 Di Martino ES, Bohra A, Vande Geest JP, Gupta N, Makaroun MS, Vorp DA: Biomechanical properties of ruptured versus electively repaired abdominal aortic aneurysm wall tissue. J Vasc Surg 2006;43:570-576; discussion 576.

29 Seo J, Choi D, Rienmueller R, Lim JG, Chang Y, Lee J: Suggestion for a new image-based aortic wall stiffness evaluation technique: arterial wall stiffness index. Int J Cardiovasc Imaging 2009;25(suppl 1):83-94.

30 Koivistoinen T, Koobi T, Jula A, Hutri-Kahonen N, Raitakari OT, Majahalme S, Kukkonen-Harjula K, Lehtimaki T, Reunanen A, Viikari J, Turjanmaa V, Nieminen T, Kahonen M: Pulse wave velocity reference values in healthy adults aged 26-75 years. Clin Physiol Funct Imaging 2007;27:191-196.

-31 Suever JD, Oshinski J, Rojas-Campos E, Huneycutt D, Cardarelli F, Stillman AE, Raggi P: Reproducibility of pulse wave velocity measurements with phase contrast magnetic resonance and applanation tonometry. Int J Cardiovasc Imaging 2012;28:1141-1146. 